\title{
Claroscuros del corazón: Kokoro (Natsume Sōseki-Kon Ichikawa)
}

\section{Chiaroscuros of the heart: Kokoro (Natsume Sōseki-Kon Ichikawa)}

\author{
Laura Montero Plata \\ Universidad Autónoma de Madrid \\ lmonteroplata@gmail.com \\ ORCID ID: 0000-0003-1510-4913
}

Resumen: Kokoro es una de las obras maestras de la literatura japonesa. Escrita en primera persona y en dos tiempos narrativos distintos, la novela más aclamada de Natsume Sōseki entabló un diálogo con la propia realidad de su tiempo; la melancolía, la ambigüedad y la ironía se mezclaron en un texto de gran sutileza. Cuando el cineasta Kon Ichikawa decidió rodar Kokoro, el desafío fue múltiple: ¿cómo adaptar una obra con dos voces y dos tiempos narrativos? ¿cómo capturar la esencia de un Japón que estaba desapareciendo con la llegada de la era Taishō? Dando una vuelta de tuerca a la fuente literaria, Ichikawa centró su versión en la representación del tabú y puso su largometraje en diálogo con los discursos teóricos que han circulado sobre Kokoro.

Palabras clave: Novellieri, Inquisición, Traducción, siglo XVI.

\begin{abstract}
Kokoro is one of the masterpieces of Japanese literature. Written in first person and in two different narrative times, Natsume Sōseki's most acclaimed novel initiated a dialogue with the reality of his time; melancholy, ambiguity and irony were blended in a text of great subtlety. When filmmaker Kon Ichikawa decided to shoot Kokoro, the challenge was multiple: how to adapt a work with two voices and two narrative times? How to capture the essence of a vanishing Japan with the arrival of the Taishō era? Giving a twist to the literary source, Ichikawa focused his version on the representation of taboo, and put his film in dialogue with theoretical discourses circulating around Kokoro.
\end{abstract}

Key Words: Novellieri, Inquisición, Traducción, siglo XVI. 
«Las palabras no son solo vibraciones en el aire. Tienen más poder que eso, pueden agitar con fuerza el corazón de los hombres y sacudirlos»

(Natsume Sōseki, Kokoro).

En un contexto cambiante y desgarrador en el que Japón sufría una transformación acelerada por la apertura del país a las influencias extranjeras, la sociedad pagó el alto precio de pasar precipitadamente de las viejas tradiciones a una repentina modernización que se inició con la llegada de la era Meiji (1868-1912). La clase intelectual, que se había cultivado en los clásicos chinos y en los preceptos confucianos, se vio inmersa en una fuerte crisis de valores cuando más tarde fue enviada a Occidente a aprender sus usos y costumbres y así poder aplicarlos al modelo de crecimiento japonés. Toda una generación fue testigo de cómo la psique colectiva se iba resquebrajando para dar paso a un nuevo Japón, cuyos rasgos definitorios serían enmarcados por la muerte del emperador Meiji el 30 de julio de 1912 y la inauguración del periodo Taishō.

En este contexto de estrés social comenzó Natsume Sōseki a escribir Kokoro. Obra de gran sensibilidad y culmen de su talento literario, la novela se convirtió en un retrato de ese cambio, en una reflexión sobre la ansiedad vertida en sus conciudadanos y una exploración pormenorizada de los intrincados recovecos del corazón humano. Dejó definitivamente atrás el humor y la ligereza que habían caracterizado sus primeros trabajos para indagar en el coste emocional que supuso el paso trepidante de los códigos confucianos al individualismo occidental (McKinney, 2010: VII). En su certera introducción a la traducción inglesa de Kokoro, Meredith McKinney subraya con insistencia la necesidad de conocer el contexto de la obra para poder apreciar en su totalidad la importancia y la repercusión del último trabajo de Sōseki publicado en vida:

Sōseki se centró cada vez más en la experiencia prototípica de sus contemporáneos, una que él mismo sentía intensamente: la necesidad de desarrollar un moderno, individual sentido de uno mismo y de lidiar con los problemas resultantes de este nuevo yo de Meiji: aislamiento, alienación, egotismo y una profunda dislocación de su herencia cultural y moral. Sōseki buscó cada vez más retratar para sus lectores no solo las turbulencias de su súbito mundo cambiante sino también los dilemas y sufrimientos de la psique contemporánea (McKinney, 2010: IX) ${ }^{1}$.

\footnotetext{
${ }^{1}$ Todas las traducciones de textos en inglés son propias.
} 
Si bien el texto arroja un análisis certero de su realidad circundante, Kokoro también se puede leer como una exorcización de algunos de los demonios de su autor, puesto que parte de su biografía y de sus propios fantasmas se cuelan en el pasado y en la filosofía vital de sus personajes.

\section{UN YO EN TRANSICIÓN: FONDO Y FORMA DE KOKORO}

El 30 de marzo de 1914, casi dos años después de la muerte del emperador Meiji y de la inauguración de Taishō, Natsume Sōseki escribió a su editor de Asahi Shinbun - uno de los periódicos de tirada nacional más importantes del país- para anunciarle que estaba trabajando en una serie de relatos cortos que se englobarían bajo el título general de Kokoro (Nathan, 2018: 219). El germen pronto viraría hacia otros vericuetos narrativos para convertirse en una gran novela por entregas, dividida en tres partes y centrada en dos historias narradas en primera persona y en dos tiempos narrativos separados. La estructura resultante quedó establecida en dos secciones contadas en presente - "Sensei y yo" y "Mis padres y yo"-, es decir, en la era Taishō; y una última parte titulada "El testamento de Sensei", que, aunque escrita desde el presente, se vuelca en la reconstrucción de un pasado atormentado acontecido en la era Meiji.

Publicada entre abril y agosto de 1914, la novela posee la cualidad de que su último tramo fue concebido en primer lugar. Esto implica que solo conociendo los eventos relatados en "El testamento de Sensei", el lector es capaz de comprender el extraño comportamiento de Sensei en las partes relatadas con anterioridad. Si bien este "no saber" en torno al que juega la novela hace que la melancolía, la angustia y la anticipación se conviertan en los ejes que marcan el ritmo de Kokoro, también lo es que no se pueda arañar la profundidad de la dura reflexión de la obra y su ironía subyacente sin tener en cuenta el oscuro secreto de Sensei. No obstante, este es un sacrificio del que el autor fue plenamente consciente puesto que su «técnica narrativa permite que el lector progresivamente vaya ahondando en la personalidad de sensei $^{2}$ a través del verdadero viaje de despertar del "yo" que realiza el joven estudiante en pos del misterio de sensei» (Rubio, 2009, s. pág.). Sin embargo, en este despertar del "yo", se terminan reproduciendo los mismos errores del pasado, puesto que si Sensei termina traicionando de forma fatal a su amigo de la infancia, el discípulo ignorará los preceptos de la piedad filial para ir en busca de Sensei, dejando atrás a un padre moribundo en su lecho de muerte.

Este "yo" en torno al que orbita la novela es, en realidad, un "yo" múltiple que alude a varias significaciones. Por un lado hace referencia al

\footnotetext{
${ }^{2}$ En cursiva en el original.
} 
protagonista del relato a quien simplemente conocemos bajo el nombre de "Yo". Por otro, invoca a la primera persona narrativa, dado que tanto Yo como Sensei utilizarán este modo para relatar los acontecimientos. Sin embargo, estos no serán sus únicos usos ya que ese yo también abarca tanto a Natsume Sōseki como autor -Kokoro está atravesada por pinceladas de su propia biografía- como al propio género literario al que se adscribe la novela: watakushi shōsetsu, que podríamos traducir como "la novela del yo". En última instancia también nos encontramos con este "yo" como término que engloba la noción de individualismo occidental, que en el mundo de Kokoro se traducirá como puro egoísmo.

Con todo, Kokoro apela a un "yo" universal; el relato de traición, silencio y expiación es enmarcado por Sōseki en un periodo muy concreto de convulsión cultural, pero sus premisas emocionales traspasan cualquier frontera. Este es también el motivo por el que los protagonistas carecen de un nombre propio, hecho que les permite convertirse en un arquetipo y en una realidad mucha más compleja y profunda que ellos mismos.

En la trama propuesta por la novela Yo conoce a Sensei un día en la playa de Kamakura y queda fascinado por su presencia. A partir de este encuentro se forja una suerte de amistad entre ambos en la que el discípulo se empeña en intentar conocer el alma de su maestro mientras este se resiste a salir de su aislamiento emocional. Tras múltiples charlas y encuentros compartidos, Sensei promete desvelar su pasado en un futuro próximo, pero la enfermedad del padre de Yo hará que el joven deba regresar al hogar familiar. En este lapso temporal, el emperador Meiji cae enfermo y fallece. El país es sacudido por la desgracia, pero el impacto será mucho mayor en la generación de Sensei (la misma que la del padre de Yo) cuando llegue a sus oídos que el General Maresuke Nogi y su mujer han cometido junshi ${ }^{3}$ para limpiar la culpa de haber fallado al emperador durante la rebelión de Satsuma en la guerra ruso-japonesa en 1877 . En el mundo de la novela y en el de Sensei, este será el último gesto de una época que muere, de una era -la Meiji- que se acaba de extinguir; de un periodo en el que el honor y la responsabilidad social estaban por encima del individualismo. El anuncio de la muerte de Nogi termina precipitando la propia muerte de Sensei, quien, en un último acto de expiación, se suicida para morir junto con los últimos vestigios de unos valores que su país está abandonando al abrazar la industrialización y la era Taishō. Es en este momento cuando escribe una larga carta a Yo, en la que revela la razón de su autoinfligida alienación social. Cuando era estudiante, Sensei vivió en la misma casa con K, su amigo

\footnotetext{
${ }^{3}$ Autoinmolación del siervo tras la muerte de su señor.
} 
de la infancia, el tercer personaje protagonista sin nombre de la novela. El destino quiso que ambos terminaran enamorándose de la hija de su casera. Sensei había llevado su amor en secreto y cuando su frágil y tímido amigo le confesó sus afectos, Sensei temió perder el afecto de la joven en pos de su amigo. Movido por el miedo y el egoísmo, Sensei pidió la mano de la joven a espaldas de K. Cuando este descubrió días después la traición de su amigo, se quitó la vida. Sensei cargará con la culpa el resto de su vida y su matrimonio se verá empañado por este trágico desenlace, aunque su mujer Shizu nunca llegará a descubrir la razón de la infelicidad de su marido. El objetivo de Sensei al depositar este peso sobre los hombros de Yo no es la búsqueda de una mera expiación, sino que espera que el joven pueda aprender de sus errores, sin ser consciente de que esa misma carta será la culpable de que Yo incurra en otra traición al dejar a su padre en su lecho de muerte mientras él se dirige a Tokio para intentar impedir fútilmente el suicidio de su maestro.

\section{TESTAMENTO LITERARIO}

Con la escritura de Kokoro y la presentación de los diferentes "yos" en ella desplegados, Natsume Sōseki quiso hacer, como ya hemos visto, una reflexión sobre el cambio cultural que se había gestado en Japón a partir de la fisura identitaria resultante de la fricción en la tradición cultural y la llegada de la mentalidad occidental. Una cavilación que, por su parte, también centró parte de la obra de Mori ōgai, otro célebre literato coetáneo del autor. En el caso de Sōseki, esta meditación le llevó a perfilar una filosofía vital a la que daría vida a través de sus personajes, convirtiendo su segunda trilogía - Más allá del equinoccio de primavera (1912), El caminante (1913) y Kokoro (1914) - en su propio testamento sobre la condición humana y su lucha interior:

Hacia esos años (1910-1916), Sōseki empieza a dar cuerpo a su ideal filosófico plasmado en la frase «seguir al Cielo y abandonar el yo» (sokuten kyoshi). Esta frase no aparece en ninguna de sus obras, pero figura en la relación de sus conversaciones con discípulos. Pues bien, en estas novelas va a darse expresión artística al drama librado dentro de los protagonistas que se debaten entre la imposibilidad de seguir al Cielo y la de abandonar su egocentrismo. La naturaleza del hombre y su destino, y no tanto la sociedad, que pasa ahora a un segundo plano, son los temas dominantes (Rubio, 2009, s. pág.).

El ideal pasa a ser la premisa de la novela pero también se convierte en la propia filosofía de $\mathrm{K}$, un hombre profundamente religioso y frugal que decide quitarse la vida para despojarse del yo, es decir, para abandonar definitivamente sus anhelos egoístas y terrenales. No será este el único rasgo de Sōseki que se filtre en el personaje de K; no en vano nos encontramos ante 
un watakushi shōsetsu. Si de su traducción podemos inferir que se trata simplemente de un género construido a partir de la narrativa en primera persona, "la novela del yo" no se limita a establecer la procedencia de la voz narradora sino que también apunta a que nos encontramos ante una prosa que encierra tintes autobiográficos. De hecho el término también podría traducirse como "novela personal", dando una noción más acertada de su esencia. De carácter naturalista, este subgrupo de novelas -muy en boga en el Japón de los años 20- se centra en las circunstancias vitales de sus protagonistas, a través de una serie de hechos que se convierten en reflejo de aspectos de la propia vida de su escritor y que, por tanto, otorgan a la obra un aura de sinceridad y de validación epistemológica (Mostow, 2003: 32).

Estos rasgos anidarán principalmente en el personaje de $\mathrm{K}$, aunque eso no quiera decir que la personalidad de Sensei no se vea también impregnada de determinadas facetas de su creador. Al uso del lema vital que $\mathrm{K}$ enarbolará, Sōseki añade un pasado teñido por el desarraigo familiar. En la novela Sensei relata que K, hijo de un monje budista, fue dado en adopción a la familia de un médico cuando cursaba la Secundaria. El joven fue enviado a Tokio para seguir los pasos laborales de su nuevo progenitor, aunque $\mathrm{K}$ pronto siente la llamada religiosa y se consagra al estudio del Budismo. A espaldas de su familia inicia un periodo de intensa inmersión espiritual hasta que finalmente es descubierto y ambas familias, la consanguínea y la adoptiva, lo repudian y abandonan a su suerte.

Las adopciones -normalmente de varones- como un acuerdo privado entre familias era una práctica muy habitual en Japón. Este acuerdo nunca afectaba al hijo primogénito y solía ser implementado como medio para preservar el apellido de la familia de adopción y como vía para asegurar el porvenir de los hijos segundones; a cambio de adquirir el nombre de familia de acogida y de preservar así el linaje de su nuevo hogar, el hijo adoptivo se aseguraba la promesa de heredar y, por ende, de un futuro mejor (Nakano, 2018: 79). Esta costumbre no fue ajena al propio Sōseki, quien fue entregado en adopción a una pareja local sin hijos. El arreglo solo duraría unos pocos años y el pequeño Natsume sería aceptado a regañadientes de vuelta por su familia de origen, junto a su madre adoptiva, tras el divorcio de la pareja de acogida. A este ya de por sí traumático evento hubo de sumarse la codicia de su padre adoptivo, que chantajeó a Sōseki cuando ya se había convertido en un aclamado autor para que lo mantuviera económicamente, argumentando una piedad filial de la que se sentía merecedor (Etō, 1965: 605).

No sería este el único personaje de Kokoro que absorbería parte del recorrido vital del escritor. El propio Sensei, también un intelectual 
educado en un sistema sino-japonés y luego enviado al extranjero ${ }^{4}$, muestra un idéntico desapego de sus obligaciones sociales. Sensei tiene la misma edad y le otorga una importancia capital a esa moral educativa de la vieja escuela; de igual modo, ambos se verán desgarrados por la asimilación del individualismo occidental, hasta el punto de sentirse atenazados continuamente por conflictos interiores irresolubles (McKinney, 2010: VIII). El hecho de que Sensei carezca de una profesión activa también podría sumarse a la lista, a pesar de que Sōseki sí se ganaba la vida ejerciendo de novelista. Natsume Sōseki perteneció a una de esas primeras generaciones japonesas enviadas al extranjero, en su caso a Londres, para absorber todo el conocimiento posible y, a su regreso, aplicarlo al crecimiento y progreso de su país. Sin embargo, tuvo una estancia miserable y solitaria en Inglaterra $y$, aunque a su vuelta encontró trabajo en el departamento de Literatura de la Universidad Imperial de Tokio, nunca logró disfrutar de este puesto. Tanto fue así que cuando alcanzó éxito como autor, no dudó en aceptar la oferta de Asahi Shinbun de publicar sus trabajos futuros y abandonó definitivamente su plaza en la universidad en 1907:

Para el estado Meiji conceder prestigio social al compromiso abnegado por el éxito material era una forma de movilizar a la gente para contribuir a la construcción de una industria japonesa moderna. Los académicos han argumentado que los personajes de Sōseki desafían los valores oficiales al servicio público y el sacrificio privado. En lugar de establecerse en el mundo exterior por el bien de la nación, se establecen en su kokoro, un sitio en el que la religión y el individuo desafían los valores oficiales de lo secular y del estado (Naito, 2010: 25).

Aunque Sōseki sí cumplió sus obligaciones sociales acogiendo discípulos e intelectuales de su casa, este rol no lo ejercería desde una tribuna universitaria dado que su papel en el panorama cultural de la época lo entablaría desde el salón de su casa, en claro antagonismo con Sensei, que mantiene su hogar ajeno a todo contacto con el exterior. El trasiego de visitas era tal que su trabajo se vio afectado y el autor se vio obligado a inaugurar "la reunión de los jueves", una cita semanal en la que colegas y discípulos eran conminados a visitarle e intercambiar ideas (Cordobés, 2014: 13; Nathan, 2018: 117-18).

\footnotetext{
${ }^{4}$ Esta asunción se puede extraer del apartado en el que Yo relata cómo conoció a Sensei, dado que explica que la primera vez que lo vio iba acompañado de un extranjero (un hecho bastante insólito en la época).
} 


\section{JUN ETŌ Y LA GLORIFICACIÓN DE LOS VALORES PERDIDOS}

Kokoro ha vertido ríos de tinta y se ha convertido en uno de los libros capitales de la literatura japonesa y en la obra maestra de su creador. A pesar de su complejidad y de los diferentes prismas de análisis que arroja, en un primer momento el entusiasmo se concentró casi exclusivamente en la tercera parte, en el testamento de Sensei. El texto, bien es cierto, se puede leer como un trabajo completo en sí mismo, pero al desechar la reflexión de Yo y eliminar el contraste narrativo entre estas dos generaciones se pierde una parte capital del mensaje para concentrarse en la glorificación de un pasado perdido definitivamente. Para Jun Etō, especialista en la obra del autor, con la muerte de Natsume Sōseki y de Mori ōgai se perdió el sentido de la misión nacional, de la responsabilidad social y de la identidad cultural. Etō incurrirá así en la nostalgia de ese japonés que ha muerto con Meiji en su análisis de la novela:

Pero la muerte del emperador Meiji y el junshi del General Nogi hicieron que [Sensei] se diera cuenta de que el espíritu de Meiji no había muerto por completo en su interior. Ahora la sombra del conjunto de la estructura de valores de esta gran era emergió en su totalidad de su atormentado pasado, sonriéndole como el fantasma de un ser querido. Tal vez el fantasma le susurró: "Ven a mí". Y Sōseki respondió, indicando a través del protagonista de su novela que una parte de sí mismo había muerto con el fallecimiento de la era Meiji. Por lo tanto, Sōseki escribió Kokoro para dejar claro que estaba del lado del fantasma de la tradición ética, pero de alguna forma universal -la ética del estoico templado o anti-egoísmoaunque plenamente consciente de que todo el sistema de valores de la era Meiji se había desmoronado mucho antes de la muerte del Emperador, y que una nueva era emergía de este caos, una era en la que la afirmación descontrolada del ego no se consideraría un acto de fealdad sino el privilegio de las generaciones más jóvenes (Etō, 1965: 619).

En su libro Perversion and Modern Japan: Psychoanalysis, Literature, Culture, coeditado junto a Nina Cornyetz, J. Keith Vincent apunta el conservadurismo de la postura de Etō, no solo porque revisita el trabajo de Sōseki desde un halo de nostalgia, sino porque transforma el testamento de Sensei en un valor absoluto que dista mucho del manuscrito original. Desde esta interpretación, Vincent explica que los académicos más conservadores despojaron a Kokoro de la meditación que Yo hace sobre la vida de su mentor para centrarse solo en la tercera parte del libro, privilegiando solo una voz en primera persona: la de Sensei, la del pasado. De este modo se eliminaba la posibilidad de una interacción dinámica entre los dos narradores, transformando Kokoro en un libro de texto moral atemporal (Cornyetz y Vincent, 2010: 224). Como resultado, lo que se busca privilegiar es un discurso en el que se concluye que Sensei se quita la vida por haber 
traicionado a su amigo $\mathrm{K}$, con el consiguiente ensalzamiento de los valores tradicionales frente al cambio de los tiempos. Sin embargo, dicha interpretación arrebata a la novela toda la ambigüedad en ella contenida y desestima uno de los grandes rasgos literarios de Sōseki: la escritura de un evento crucial de forma casual, sin elaborarlo pormenorizadamente y sin hacerlo evidente (Kawana, 2018: 78).

Posteriormente llegarían otras teorías que refutarían esta interpretaciones: unas más radicales -sobre todo las expresadas por Yōichi Komori, como veremos más adelante- y otras más en sintonía con las lecturas más convencionales y aceptadas. Cuando el cineasta Kon Ichikawa recibió el encargo de hacer una adaptación cinematográfica de Kokoro, la novela todavía no había entrado a formar parte de la enseñanza obligatoria; lo haría un año después, en 1956, de la mano de la editorial Shimizu Shoten, que seleccionó un fragmento de la primera parte. No sería hasta los años sesenta cuando se preferenció el estudio de "El testamento de Sensei", una práctica que se prolonga hasta nuestros días (Kawana, 2018: 224, n. 4). Por lo tanto, cuando Ichikawa comienza su adaptación, la lectura de la obra seguía adscrita al triángulo amoroso entre Sensei, K y Shizu, y a la nostalgia de los valores perdidos.

\section{LA ADAPTACIÓN LITERARIA Y SUS RESIGNIFICACIONES}

En el periodo de esplendor del género bungei eiga -adaptaciones cinematográficas de grandes obras de la literatura-, Kon Ichikawa recibió el encargo de la productora de Nikkatsu de dirigir Kokoro. Esta sería la segunda de las tres extrapolaciones a la pantalla de clásicos literarios que el cineasta haría para la compañía, aunque la más famosa de ellas y la que le daría el reconocimiento internacional sería El arpa birmana (Biruma no tategoto, 1956) tras su triunfo en el Festival Internacional de Cine de Venecia. Procedente del mundo de la ilustración y de la animación, el cineasta se distingue por la extensa profusión de su filmografía - que incluye más de setenta títulos- $\mathrm{y}$ su eclecticismo. El propio creador se veía más a sí mismo como un artesano que como un autor, y saltaba de un género a otro sin distinción. La falta de tropos visuales reconocibles o de temas recurrentes así lo indica pero, como bien apunta el especialista en cine japonés Jasper Sharp, Ichikawa poseía un gran talento pictórico que le permitía crear imágenes sumamente poderosas (Sharp, 2018, s. pág.).

Sin ser su obra más brillante, su versión de Kokoro destaca por ser una adaptación sumamente fiel donde se replican diálogos sacados de la novela pero que, a su vez, cambia de forma definitiva las interpretaciones extraídas de la fuente. Quizá esto se deba justamente a que Natsume Sōseki se esforzó por mover a sus personajes en las tormentosas aguas de lo impreciso, mientras que Ichikawa elige un enfoque bien definido que pasa por centrar 
la trama en la atracción homosexual a la que se ven sujetos los protagonistas de su propuesta. Sin embargo, los cambios empiezan mucho antes, en la propia concepción del guion que introduce tres modificaciones críticas en el desarrollo de los hechos: el abandono de la narración en primera persona, la introducción de nombres propios y la desestimación de la estructura tripartita original en aras de una suerte de relato "cronológico". Sin ningún tipo de voz en off que nos desvele, como en la novela, el tormento interior de Yo y de Sensei, la alternativa de Ichikawa se sitúa en una distante tercera persona que acecha a los personajes y se deleita en sus silencios y en sus gestos; curiosamente el silencio juega en las dos versiones un papel capital aunque diferente: mientras que en la novela apunta oscuros secretos nunca expresados en voz alta, en el filme se recrean en desnudar emocionalmente a sus protagonistas forzando primeros planos que intentan penetrar en las almas de quienes se resisten a exponer sus emociones ante la cámara.

Con la desaparición del "yo", al que ni siquiera se le permite expresar sus cavilaciones como narrador fuera de campo, se pierden también esos "yos" diseminados a lo largo del texto de Sōseki y se desvincula del género al que estaba suscrito, a esa "novela del yo". También se desvanece la premisa formativa del texto de 1914 puesto que, como ya apuntamos con el certero análisis de Carlos Rubio, es gracias al viaje del despertar del “yo” en busca de su mentor como nos sumergiremos en la compleja personalidad de Sensei. En la versión de Ichikawa, las líneas dramáticas corren en paralelo -por más que una transcurra en el pasado-, de tal forma que el impacto devastador que provoca el testamento de Sensei en Yo no tiene ni por asomo las mismas repercusiones en la película, puesto que el crimen de Sensei ha sido revelado al espectador antes de que el estudiante abandone a su padre moribundo.

El segundo gran cambio del filme gira en torno a los nombres masculinos; si en el libro estos se mantenían como arquetipos de dos épocas -una que se desvanece y otra que acaba de consolidarse--, la película trasforma a los integrantes del relato en individuos tocados por la tragedia y los desposee de ese carácter de arquetipo de un mundo cambiante. "Yo" con la desaparición de la primera persona- se convierte en Hioki, Sensei responde al nombre de Nobuchi -aunque el estudiante siempre se referirá a él como "sensei"-; mientras que $\mathrm{K}$ pasa a ser Kaji. El distanciamiento impuesto por Sōseki se desvanece en una época, la de 1955, en la que la Nuberu Bagu - la Nueva Ola japonesa- todavía no había hecho su aparición en escena. Sin duda, la ruptura del lenguaje fílmico convencional hubiera sido la solución más idónea a este desafío en la traslación a la gran pantalla, pero el convencionalismo impuso su norma. Esto no es justificación, con todo, para defender algunas de las omisiones en las que incurre la versión de Ichikawa. El crítico Tony Ryans hace de hecho, en el libreto que 
acompaña la edición inglesa en DVD de Kokoro, una disertación sobre el cambio de significado ya desde el propio título de la película.

Kokoro es un término difícil de traducir al español puesto que la palabra encierra varios significados entrelazados entre sí; el más común de ellos es 'corazón', aunque también se refiere a 'mente', 'alma', 'espíritu', 'mentalidad', 'sensibilidad', 'emociones', 'pensamiento', etc. Este es el motivo por el que la mayoría de las traducciones han dejado intacto el título original japonés, puesto que la novela abarca el concepto en su plenitud polisémica. En su análisis sobre este título, Ryans va un paso más allá al explicar por qué Sōseki eligió su lectura en hiragana ${ }^{5}$ (こころ) en lugar de escribirlo en kanji $^{6}$ (心):

La elección de Sōseki del hiragana en este caso -el título de la película por supuesto lo imitará- tiene el efecto de difuminar el significado, haciéndolo parecer menos inequívoco y más abierto a matices semánticos y filosóficos. Para Sōseki, esto estaba asociado a su sensación de que la psique nacional japonesa estaba cambiando; vio un vacío en el kokoro de Japón causado por presiones externas de Occidente y presiones internas para asimilarlos. (En la novela, el estudiante primero ve al hombre mayor acompañado por un amigo extranjero en la playa, un significativo detalle que el filme omite). La película pone un énfasis diferente en los tiempos cambiantes, enfatizando - y validando- la intuición del estudiante de que el presente de uno está modelado por su propio pasado (Ryans, 2009: 8).

Si enlazamos el cambio de significación del título con el cambio de dirección dramática que imprime la imposición de nombres propios a los personajes concretos, la distorsión es aún mayor. No hablamos solo aquí de la eliminación de los arquetipos, sino del misterio construido alrededor del personaje de K. "Yo" como narrador principal tiene un sentido claro e inequívoco, "Sensei" es un honorífico para designar a un individuo respetable socialmente por haber alcanzado un elevado grado de maestría en su campo, que nosotros traduciríamos como "maestro". Aunque en Japón sensei suele ir precedido por el apellido de la persona a la que uno se refiere, se puede emplear a secas para designar a nuestro interlocutor. El caso de $\mathrm{K}$ es diferente. ¿Por qué Sōseki elige una consonante para referirse a él? ¿Por qué K? ¿Podría ser está $\mathrm{K}$ otra referencia a ese kokoro traicionado en torno al que se desarrolla la novela? Sin tener una respuesta clara a esta pregunta, lo que es evidente es que al otorgarle el nombre de Kaji en la película, se

\footnotetext{
${ }^{5}$ Uno de los dos silabarios que configuran el sistema de escritura japonés.

${ }^{6}$ Conjunto de caracteres de origen chino que, junto a los silabarios de hiragana y katakana, conforma la escritura del japonés.
} 
elimina nuevamente este juego de ambigüedad y, con él, buena parte del misterio que envuelve al personaje.

Con todo, el tercer cambio en la trasposición al cine será el que provoque un mayor impacto en el relato, dado que en la versión de Kon Ichikawa la tragedia queda mitigada por la reordenación de los hechos. La división en tres partes desaparece y se introduce una línea cronológica que se interrumpirá en seis ocasiones para relatar acontecimientos del pasado; el más largo será por supuesto el testamento de Sensei, que dividido en dos partes - presentación del personaje de Kaji y desarrollo de la tragedia- tiene una duración de 51 minutos. Ichikawa reordena capítulos -incluso dentro de una misma parte-, inventa nuevas situaciones y da origen a este nuevo tiempo narrativo en el que se pierde la temporalidad original, como podemos apreciar en el cuadro cronológico que rehace el tiempo narrativo de Kokoro:

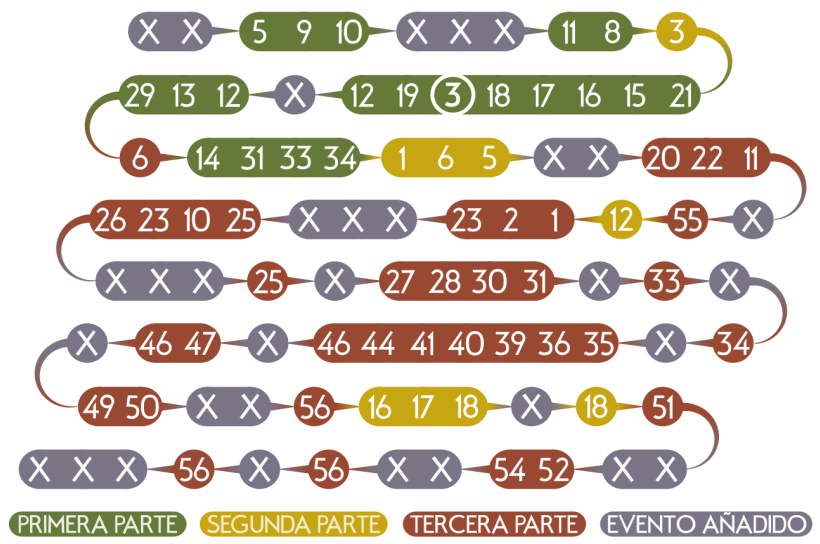

A pesar de la idea general de reorganización que nos proporciona el cuadro, conviene enumerar los distintos flashbacks que se incluyen en la película:

-Minuto 19 (capítulo 3, primera parte): Encuentro en el mar de Hioki y Sensei (episodio con el que se abre la novela).

-Minuto 26 (capítulo 6, tercera parte): Traición del tío de Sensei.

-Minuto 39 (capítulo 20, tercera parte): Introducción del personaje de Kaji y su mudanza a la posada de Sensei. 
-Minuto 51 (capítulo 23, tercera parte): Relato del triángulo amoroso entre Kaji, Nobuchi y Shizu.

-Minuto 108 (capítulo 23, tercera parte): Boda entre Nobuchi y Shizu, y vida conyugal.

-Minuto 114 (capítulo 3, primera parte): Encuentro en la playa de Hioki y Sensei (repetición).

-Minuto 115 (evento añadido): Primeras visitas de Hioki a la casa del matrimonio después del encuentro en la playa.

Si en la novela la tensión dramática surgía del desconocimiento del secreto de Sensei, en la película será la premonición de la muerte la que asentará el tono de la narración. Para lograr este efecto, Ichikawa abre la película con unos títulos de crédito contra los que superpone una escena marina. El agua ocupa casi la totalidad de la pantalla y el vaivén de las olas parece engullir los diferentes nombres que van desfilando ante nuestros ojos. El acompañamiento de cuerdas crispado asentará el tono inquietante de la cinta y anticipará el tema musical del pecado de Sensei cuando vuelva a sonar en la visita de Nobuchi al cementerio.

En la relación de los distintos flashbacks que atraviesan el metraje, el encuentro en la playa, como ya apuntaba Ryans, poco tiene que ver con los hechos expuestos por Sōseki. En primer lugar porque se omite la presencia del amigo extranjero (alusión al cambio cultural provocado por la era Meiji); en segundo, porque la intención del maestro no es en ningún momento la del suicidio; y, en última instancia, porque el encuentro entre los dos hombres permite introducir a Ichikawa el gran leit motiv de su adaptación: la homosexualidad reprimida. Un tema que, orbitando muy sutilmente sobre la prosa de Natsume Sōseki, lo empaña todo de deseos carnales no expresados en el caso del largometraje.

\section{El CONTINUO HOMOSOCIAL EN KOKORO}

Mucho se ha discutido en el ámbito académico sobre el retrato que hace el novelista de un mundo en vías de extinción en el que los jóvenes varones japoneses entablaban intensas relaciones con sus compañeros, ya fueran estas de pura índole intelectual o de carácter físico, hasta su "graduación" de este ámbito al convertirse en "hombres" como parte de un rito de paso hacia la integración social como adultos (Kawana, 2018: 86). Ya en el año 1971, el académico Doi Takeno aseveró, refiriéndose a la novela, que «ningún trabajo literario había retratado con tanta precisión la naturaleza de las relaciones homosexuales en la sociedad japonesa» (Nathan, 2018: 297, n. 19). Por su parte, J. Keith Vincent analizó el capítulo 13 de "Sensei y Yo" como representativo del punto de inflexión en el cambio histórico de la dinámica de la sexualidad hombre a hombre en Japón, dado que tanto Sensei como el propio Natsume Sōseki son miembros de la última generación que 
recordaba esa época en la que el continuo homosocial seguía siendo una costumbre para propiciar el apego masculino a través del sexo entre hombres. En la generación intermedia, entre Meiji y Taishō, el vínculo se rompería en el mencionado pasaje de la novela, lo que provocó que Yo no fuera consciente del significado de las palabras de Sensei. Vincent afirma, pues, que el mundo moderno en el que vive el joven ha eliminado la posibilidad del amor entre hombres y que de ahí surge su incomprensión (Cornyetz y Vincent, 2010: 222). El diálogo del capítulo 13 transcurre del siguiente modo:

-No tardarás en comprenderlo, aunque puede que ya lo hayas hecho. Tu corazón está inquieto por culpa del amor, ¿no es así?

Miré en mi corazón para comprobar si lo que Sensei decía era cierto, pero lo único que encontré en mi interior fue un gran vacío. No había nada allí que se correspondiera a sus palabras.

-No hay en mí ningún objeto de amor, Sensei. Créame, soy honesto con usted.

-Precisamente por eso. Si estás inquieto es porque no hay nada. ¿No lo ves? Te mueve la idea de que solo si encuentras ese objeto podrás estar en paz.

- No siento demasiada inquietud en este momento, a decir verdad.

- Si te acercaste a mí es porque sentías alguna carencia en tu alma. ¿O no?

-Es posible, pero no es amor lo que me falta.

- Es un primer paso en dirección a ese amor. Has sentido el impulso de acercarte a alguien del mismo sexo como primer paso para poder acceder a alguien del contrario.

-Pues a mí me parece que son dos cosas de naturaleza absolutamente opuesta.

—No, son la misma, créeme. Pero como yo soy un hombre, no puedo llenar ese vacío tuyo. Además, hay ciertas cosas que hacen imposible para mí ser quien tú quieres que sea. Lo lamento de veras. Acepto que la urgencia de tu inquietud te llevará un día a otro lugar, lejos de mí. De hecho, espero por tu bien que así sea. Y sin embargo...

Noté que me invadía una súbita tristeza (Sōseki, 2014: 51-52).

En la era Meiji, las relaciones de carácter homosocial en las escuelas de élite, en las universidades y en el ámbito militar eran concebidas como una práctica masculinizante. No se percibían como una actividad minoritaria, sino como una forma de conexión entre los jóvenes. En estas instituciones educativas de élite, tales lazos afectivos podían alcanzar una gran intensidad; tal fue el caso, por ejemplo, del filósofo y educador Yoshishige Abe, quien en su época de estudiante dedicó una emotiva carta a un compañero que había cometido suicidio: «Ha pasado un año desde que te perdí y, sin embargo, no pasa un día sin que piense en ti. [...] Tu cara no debería ser 
vista y, sin embargo, veo tu rostro por todas partes... Oh, ¿por qué te extraño así?» (Kawana, 2018: 86). En su libro The Uses of Literature in Modern Japan: Histories and Cultures of the Book, Sari Kawana examina esta misiva bajo el prisma de la culpabilidad expresada por Sensei en el libro de Sōseki, justificando la presencia de los fantasmas que le acechan de forma tan intensa. Con todo, estos encuentros masculinizantes formaban parte de los ritos de paso hacia la edad adulta y uno estaba destinado a "graduarse" y pasar a formar una familia heterosexual. De hecho, continúa Kawana explicando, era habitual que algunas familias - sobre todo las conformadas por viudas y sus hijas-alquilaran habitaciones a estudiantes masculinos no solo como una fuente de protección sino como un posible medio para encontrar un marido para sus hijas.

Con la apertura a Occidente durante el periodo Meiji comenzó un intenso periodo en el que el discurso sobre nanshoku (erotismo masculino) se vio reformulado, pasando de ser un fuerte y vigorizante vínculo entre amigos a convertirse en una práctica pervertida y peligrosa (McLellond, 2000: 24). El cambio en la percepción de las relaciones íntimas entre hombres fue radical, y Natsume Sōseki lo verbaliza en este pasaje de Kokoro al exponer la incomprensión de Yo antes los alegatos de Sensei.

A pesar de la fidelidad que Kon Ichikawa imprime a su adaptación de la novela, este pasaje en concreto se ve fuertemente alterado. Si el episodio en ambos casos comienza con el maestro asegurando vehementemente que el amor es un pecado, la conversación pronto cambia de rumbo en la película hacia la inquietud de Sensei por el futuro del joven, quien podría ser engañado con su herencia una vez que su padre fallezca, un doloroso trauma que el intelectual padeció en sus años de estudiante. Así, el largometraje, en vez de explorar el continuo homosocial insinuado por Sōseki, prefiere centrarse en el secreto que oculta Nobuchi. Hioki insiste en conocer su pasado alegando que lo que pasó en él ha dado forma a su presente. Nobuchi le toca el hombro y le responde que le gustaría confiar en él porque es demasiado inocente para recelar de él.

Aunque en ambos episodios se muestra un fuerte sentimiento emocional entre los protagonistas, la versión cinematográfica prefiere centrar la mostración de este continuo homosocial a través de la amistad entablada entre Nobuchi y Kaji; por un lado porque esta sí se enmarca históricamente en una época en la que estas prácticas eran habituales, pero también porque el peso del pecado de Sensei tiene tintes profundamente homoeróticos en el filme, mientras que en la novela su interpretación queda sujeta a muchos más factores. La elección de Ichikawa resulta desafiante y astuta: para la generación de Natsume Sōseki la homoerótica era una práctica aceptada, pero con la apertura a Occidente la percepción había cambiado hasta 
convertirla en un tabú. ¿Había desaparecido el nanshoku o simplemente se había ocultado de la vista pública?

Al escoger Kokoro para adaptarlo, al ser extremadamente fiel en la plasmación de escenarios, de los silencios y de la reconstrucción de la atmósfera asfixiante del texto original para luego transformarlo en un triángulo amoroso dictado por la atracción homosexual de sus protagonistas, el cineasta provoca y sacude al espectador con la representación del tabú. Sin embargo, la simplificación a la que se ve expuesta el libreto cinematográfico - por limitaciones obvias de duración narrativa- también hace que la psicología y el tratamiento implícito de la sexualidad de los personajes varíe irremediablemente. J. Keith Vincent define la sexualidad perfilada en la novela en los siguientes términos:

Kokoro, argüiré, no es ni una novela "gay" ni una novela "hetero", sino un texto que pone en diálogo dos concepciones sobre la sexualidad: uno que crea las categorías de homosexualidad y heterosexualidad como los extremos opuestos de una narrativa desarrollacional, y otro que reconoce el papel performativo de la narrativa en sí misma en la manera en la que la que la sexualidad es experimentada y entendida. El modelo desarrollacional es presentado por el primer narrador de la novela, [Yo] [...], mientras que el segundo está implícito en el texto en su conjunto (Cornyetz y Vincent, 2010: 223).

Kon Ichikawa desafía esta dualidad y se concentra en la pulsión homosexual. Como resultado, la psicología de Nobuchi se ve sustancialmente alterada originando un nuevo ente que, si bien comparte con Sensei su marcado talante atormentado, es presentado como un personaje cruel. En parte, este cambio en la actitud del personaje se debe al duro semblante del actor Masayuki Mori y al juego de claroscuros entablado por el cineasta, que suele ubicar siempre a Nobuchi en la sombra, pero también contribuye la omisión de momentos clave del pasado del protagonista.

\section{NOBUCHI VERSUS SENSEI: SEMBLANZAS DISONANTES}

El germen de esta transformación se origina en el episodio relativo a la herencia. Si bien la película cubre parcialmente este doloroso recuerdo, solo se concentra en el empeño de su tío por casarlo con su prima sin entrar en los pormenores de la traición del hermano de su padre, en quien él confía plenamente, y que llevaba una doble vida gracias a su dinero. La inocencia de Sensei queda rota en ese momento y empieza a desconfiar de las intenciones de todos cuantos le rodean; se incluirá en este grupo a la madre de Shizu, de quien sospecha que está confabulando para que tome a su hija como esposa. El joven no tiene, sin embargo, este sentimiento hacia K. Por 
su parte, como ya hemos expuesto, $\mathrm{K}$ había sido abandonado a su suerte por sus dos familias - la consanguínea y la adoptiva- y se encontraba en una situación de desamparo cuando Sensei lo acoge y lo invita a quedarse con él. Por tanto, cuando Sensei conspira contra él de la peor forma posible y guarda silencio, su traición se agrava porque él es plenamente consciente de las implicaciones de romper la confianza de quien es más cercano a uno mismo. La otra gran alteración de la versión cinematográfica es la representación de la relación que lo ata a Shizu. En la novela se establece con absoluta trasparencia que Sensei está enamorado de la joven. De hecho, su conflicto interior radica en que la admiración ciega que siente por $\mathrm{K}$ lo hace sentirse inferior y teme que este le robe el favor de la joven. Es innegable que Natsume Sōseki siempre se mueve en el terreno de la ambigüedad, por lo que ese amor expresado por Sensei se podría traducir como esa "graduación" desde el continuo homosocial hacia una edad adulta heteronormativa.

Kon Ichikawa desdeña esta idea y elimina toda mención explícita de los sentimientos de Nobuchi hacia la joven con el objetivo de potenciar la más que evidente tensión afectiva que le une a Kaji. El punto culminante de esta relación reprimida y asfixiante se produce en el viaje que ambos jóvenes emprenden a la península de Boshu; concretamente en el episodio que transcurre al borde de un acantilado. En la novela, la escena sucede en los siguientes términos:

En realidad habría preferido que fuera Ojyosan quien estuviera sentada a mi lado, en lugar de K. Era un pensamiento agradable. A veces sospechaba que $\mathrm{K}$ pensaba exactamente lo mismo respecto a mí, y entonces perdía la calma y no podía concentrarme en la lectura. Me levantaba entonces y me ponía a gritar, incapaz de expresar mis sentimientos de una forma más civilizada, como, por ejemplo, recitando un poema o cantando una canción. Lo único que podía hacer era comportarme como un salvaje. En una ocasión agarré a K por el cuello y le pregunté qué haría si lo tiraba al mar. Me respondió, sin inmutarse, que le daba igual. Ni siquiera cambió de postura. Lo solté enseguida.

Sus ánimos parecían haberse apaciguado. Al contrario que yo. Sentía que mi serenidad se desmoronaba a ojos vista. Envidiaba su aparente calma y la interpretaba como pura indiferencia hacia mí. $\mathrm{K}$ desprendía una confianza en sí mismo que me desagradaba profundamente (Sōseki, 2018: 231).

En su versión cinematográfica, la amenaza de muerte se produce de manera similar pero el contexto es completamente distinto, no solamente por la ausencia de una voz en off que nos permita conocer el discurrir del pensamiento de Sensei, sino porque segundos antes Nobuchi rescata a Kaji de precipitarse al mar cuando su gorra cae y ambos hombres comparten un 
abrazo parcial y una mirada cómplice. Nobuchi se separa con desagrado cuando es consciente de lo íntimo que se ha tornado el gesto, se coloca detrás de su amigo y le pregunta: «¿Te alegra que hayamos venido?» Kaji permanece en silencio mirando un punto indeterminado del horizonte. Nobuchi le insta entonces a que le diga en qué piensa. Cuando Kaji responde que nada, frustrado Nobuchi lo agarra por la solapa, lo inclina hacia delante y con sonrisa macabra le increpa: «¿Qué pasaría si te empujara al mar». Kaji responde «Vamos, hazlo». Nobuchi, desconcertado, se separa y emprende el camino de vuelta. Su amigo le sigue de cerca. La escena es idéntica pero las circunstancias son opuestas. El viaje prosigue e Ichikawa reproduce el intercambio verbal de Kaji con un monje de la secta Nichiren del que el joven sale profundamente decepcionado. Kaji acusa a su amigo de no ser consciente de su sufrimiento interior -conversación que también se recoge en la novela-, pero aquí Ichikawa inserta otro giro narrativo para incidir en la pulsión íntima que se ha establecido entre ambos amigos. A esta acusación, Nobuchi responde que sí sabe lo que sufre y hastiado se aleja por la extensión interminable de arena. Kaji lo sigue con pasos ligeros y lo alcanza, momento en el que ambos terminan avanzando por el terreno arenoso cogidos de la mano en un plano general en el que la arena parece engullirlos, como ya había sucedido con la escena marina en la que Hioki y Sensei se encuentran. Ambos momentos compartidos, ambas atracciones aparecen representadas en dos escenarios en los que los personajes son amenazados con ser tragados por el vacío, en clara alegoría al tabú que se deja entrever pero que nunca se explicita. Señal inequívoca de la carga metafórica de esta secuencia la encontramos en el póster promocional japonés del largometraje, donde la escena ocupa dos tercios de la imagen y sirve de contraste a un retrato del matrimonio.

No quiere esto decir que la novela de Natsume Sōseki esté exenta de pasajes donde se deje entrever la intimidad instalada entre los personajes protagonistas, aunque, en su caso, esta sea apreciada de una forma más intensa en las diatribas entre Yo y Sensei, como se observa en un pasaje inicial del testamento del maestro:

A veces te sonreía. A veces atisbaba un mohín de insatisfacción en tu cara. Mientras tanto, me presionabas para que desplegara mi pasado como si fuera una pintura en rollo. Te respeté por ello porque mostrabas la clara determinación de extraer vida de mí, de beber la sangre caliente que brotaba de mi corazón. Entonces yo aún estaba vivo. No quería morir. En lo más profundo de mi ser me dije que un día satisfaría tu deseo y fue así como me quité toda la presión de encima. Ahora sí, ahora voy a abrirte mi corazón para verter mi sangre sobre ti. Me daré por satisfecho si cuando deje de latir, una nueva vida ha arraigado en tu pecho (Sōseki, 2018: 169). 
Sin embargo, el reproche velado abandona tal condición en el largometraje para dar paso a una increpación mucho más explícita en que la víctima principal de estas pulsiones, Shizu, acusa a su marido de tener con Hioki una relación que va más allá de la amistad:

- Shizu: ¿Cuándo le mandaste el telegrama? Has debido escribirle. Si no, no te hubiese mandado este telegrama. ¿Cuándo se lo mandaste? ¿Por qué le has llamado? ¿Qué quieres de Hioki?

-Nobuchi: Shizu...

-Shizu: ¿Qué me ocultas?

-Nobuchi: No escondo nada.

-Shizu: ¡Mientes! ¿Por qué no puedo saberlo? Lo que pasa contigo y con Hioki, lo que pasó contigo y con Kaji.

-Nobuchi: ¿Con Kaji?

- Shizu: Eso es. Kaji era tu mejor amigo. Después de su muerte ni siquiera podía mencionar su nombre. ¿Por qué me lo prohibiste?

-Nobuchi: ¡Shizu, por favor, no digas eso!

- Shizu: Hasta ahora nunca he dicho nada. Y ahora con Hioki... Él es nuestro único amigo. Estaba feliz por ti pero está pasando lo mismo de nuevo.

-Nobuchi: ¿Entre Hioki y yo? Shizu, no tengo ni idea de lo que hablas.

- Shizu: Por favor, no me mientas. Algo pasa entre Hioki y tú de lo que no sé nada. Soy tu mujer; he estado a tu lado 13 años. ¿Amas más a tus amigos que a mí? Quiero saber lo que hay dentro de tu corazón.

-Nobuchi: Estás confundiendo erróneamente a Kaji y Hioki. Sabes de sobra quién significa más para mí.

(Nobuchi sale de la habitación y da la conversación por terminada).

La pérdida de tal sutileza en el filme se debe, como ya hemos apuntado, a la carencia de una voz narrativa, a la reordenación de los eventos y al claro enfoque homoerótico de la propuesta de Ichikawa, pero, sobre todo, a la simplificación de la psicología de su protagonista, que pasa de ser un hombre contrariado por sus demonios a convertirse en un hombre que parece incapaz de amar. El investigador Paul Standish argumenta en su análisis de la cinta que la dificultad para entender las motivaciones de Nobuchi y su patente crueldad dan origen a una lectura en términos de represión -que incluye la represión homosexual-y que apunta a la impotencia y al sadismo (Standish \& Saito, 2012: 2). Tony Ryans, por su parte, señala que la relación entre Nobuchi y Hioki no se puede ubicar en los parámetros de una relación tradicional entre maestro y alumno, dado que este último parece más interesado en averiguar por qué su mentor se niega a entablar relaciones sexuales con su mujer (Ryans, 2009: 11). Aunque en ningún momento de la película o del libro se hace mención a este hecho, sí que podría extraerse tal conclusión de una escena concreta del largometraje de Ichikawa en la que Shizu, Nobuchi y Hioki conversan en el jardín de la casa del matrimonio. 
Shizu confiesa que le hubiera encantado tener hijos. Ante la sentida afirmación de la mujer, su marido replica que eso jamás sucederá. Cuando el estudiante, sorprendido, le pregunta por qué dice eso, Sensei responde con una risa sardónica que es un castigo divino.

Si bien esta escena replica palabra por palabra el texto de Sōseki, la película se sitúa en oposición a la fuente a la hora de construir el personaje de Shizu. La joven es el eje en torno al que se construye el metraje -con un primer plano de ella inicia y concluye la narración-, pero ella no es el objeto de deseo de la trama, como sí sucede en la obra escrita. La coquetería de Shizu en la adaptación es estéril puesto que Nobuchi nunca se muestra alterado por la posibilidad de que ella esté intentando acercarse a otro hombre. Lo que irrita sobremanera al maestro es que está perdiendo la atención de Kaji. Sari Kawana destaca que el cambio de dirección en el comportamiento de los personajes y, sobre todo, en el caso de Shizu, tiene el claro objetivo de justificar la posterior obsesión de Sensei con Kaji una vez que ha conseguido casarse con la joven y este ha muerto. Sin la alteración del encuentro bajo la lluvia, cuando Nobuchi muestra su alivio ante la confesión de que ella había forzado el paseo con Kaji, el comportamiento de Nobuchi hubiera sido incomprensible para el espectador (Kawana, 2018: 88).

\section{SHIZU: HACIA UNA LECTURA HETERONORMATIVA}

El cambio de actitud de Shizu en la película - que pasa de un ser puro y ajeno a todo el drama interior de su marido a una mujer frustrada que vive bajo la sombra de la sospecha - también se podría interpretar bajo otro prisma por el que Ichikawa utiliza al personaje femenino para plasmar en el filme el paso de ese estadio homosocial -entre Hioki y Nobuchi- hacia la "graduación" del estudiante al entablar una relación heteroformativa junto a la viuda de su mentor. Analizada bajo esta perspectiva, la versión de Kokoro de Kon Ichikawa se adelanta tres décadas a la controvertida relectura que el académico Yōichi Komori hizo de la novela de Natsume Sōseki en 1985.

Nacido en Tokio en 1953, Komori perteneció a ese grupo de estudiantes que leyeron y analizaron Kokoro siguiendo la interpretación más conservadora ofrecida por Jun Etō: aquella que incidía en la glorificación de un pasado y de unos valores perdidos - los de la era Meiji- y que propició que en las escuelas solo se estudiara la tercera parte de la novela, eliminando todos los matices y juegos sarcásticos que Sōseki había establecido a partir de la voz de Yo. Además de por las claras limitaciones que resultaban de convertir "El testamento de sensei" en una novela en sí misma, Komori se sintió frustrado por el final abierto de Kokoro. Sōseki no ofrecía ningún tipo de cierre para los otros protagonistas de la novela: Yo y Shizu. El novelista les impidió literalmente pasar página. Yo se había quedado suspendido en un tren eterno de vuelta a Tokio mientras lee el testamento de su amigo, y 
tampoco sabemos nada de la situación de la nueva viuda, una vez que su marido se ha quitado la vida sin permitirle entender el motivo de la infelicidad que se había instalado desde el inicio de su matrimonio.

A Komori, las explicaciones de sus profesores le parecían manidas consignas y achacaba sus interpretaciones a una profunda carencia de imaginación, puesto que la pluma del novelista se caracterizaba por esa ambigüedad narrativa que ya habíamos apuntado con anterioridad. Los libros de texto habían convertido Kokoro en una obra «que funciona como un mecanismo de la ideología nacionalista centrado en torno a los valores patriarcales de la "ética", la "espiritualidad" y la "muerte". Los jóvenes lectores son forzados a arrodillarse ante la muerte "ética" y "espiritual de “Sensei”» (Cornyetz y Vincent, 2010: 224). Con la publicación en 1985 de "Kokoro wo seisei suru hāto", Komori asentó la polémica con una lectura extremadamente transgresora del texto de Sōseki. El crítico centraba su análisis en el monólogo de Yo y en las lecciones que este extraía del testamento y del suicidio de su mentor. En su minucioso examen de la obra, el teórico japonés llegó a la conclusión de que Yo termina casándose con Shizu y dándole un hijo. Esta lectura propiciaba una conclusión más satisfactoria y venía a confirmar que Yo sí aprendía del testamento de Sensei. Con esta relectura, Komori pasaba de un análisis nacionalista en clave homosocial a otro que se orientaba hacia la llegada de una modernidad japonesa asociada con la heterosexualidad (Nathan, 2018: 228).

Pese a que son varios los argumentos que llevan a Komori a postular esta hipótesis, nosotros nos centraremos en dos. El primero, ausente en el filme, viene marcado por una dualidad creada por Sōseki a través de sus dos primeras personas narrativas y que está relacionada con la presentación de sus personajes. Por un lado, cuando Yo comienza la narración se refiere a su maestro en los siguientes términos: «Siempre lo llamé Sensei» (Sōseki, 2018: 19). Sensei, por su parte, hace la primera mención directa a su amigo de la infancia con la frase «A partir de ahora le llamaré K» (Sōseki, 2018: 208). A través de estas dos introducciones, que se duplican y se miran como en un espejo, Komori atisba una circularidad de la historia en la que, igual que Sensei toma la delantera a K y conquista a Shizu, Yo tomará el papel de marido una vez que Sensei cometa suicidio, replicando de este modo acciones, comportamientos y suicidios. El segundo argumento, mucho más sutil, pasa desafortunadamente desapercibido en la traducción de la novela

\footnotetext{
${ }^{7}$ Komori establece un juego de palabra entre el término japonés kokoro y el anglicismo häto (heart). Dado que kokoro se puede interpretarse de múltiples formas, el título de su ensayo podría traducirse al español como "El corazón que alienta el alma" o "El corazón que alienta el espíritu”.
} 
y de la película, aunque en el japonés original Ichikawa reproduzca de forma fiel el diálogo de Sōseki. En una cena en la casa del matrimonio, Nobuchi pregunta a su mujer qué haría si él muriera. Tras un intercambio verbal en el que ambos debaten quién podría morir antes, Shizu claudica y concluye mirando a Hioki: «Dō suru te? Shikataganai ha... Ne, anata» («Que qué haría... No podría hacer nada al respecto, ¿verdad, querido?»). El uso que tanto Sōseki como Ichikawa hacen de anata en este contexto provoca la inmediata extrañeza. Por más que en sentido estricto anata se traduzca como 'tú', el uso más común en japonés se destina a las mujeres casadas que lo emplean como apelativo cariñoso al hablar con sus maridos. A pesar del tono jocoso de Shizu en este intercambio verbal, el hecho de que se refiera a Hioki como anata en un contexto hipotético en el que su marido ha muerto, permite abrir la puerta a una interpretación en la que el joven ha tomado el lugar del difunto. Esta es sin duda la lectura de Yōichi Komori (Nathan, 2018: 226). Kon Ichikawa no llegará tan lejos en su adaptación pero permite al espectador interpretar su versión en unas claves parecidas. En primer lugar porque la película se centra en la pulsión homosexual establecida entre Nobuchi y Kaji -y no en la de Hioki y su mentor-; en segundo, por la inclusión de este episodio de la novela; y en tercera instancia, quizá la más relevante, porque el cineasta también se muestra insatisfecho con el final de la novela y ofrece un cierre alternativo. En él, Hioki vuelve a Tokio y se dirige a casa de Sensei. Su maestro ya ha cometido junshi, y le recibe una compungida Shizu con lágrimas en los ojos. Cuando en un arrebato la mujer se aproxima a él, Hioki cae de rodillas y se disculpa por no haber salvado a su marido. Ella llora y se seca las lágrimas, mira hacia la calle y se dirige a cerrar la puerta para darles privacidad, dejando a Hioki dentro de los confines de su hogar.

La película abre y cierra, pues, con un primer plano de Shizu, puesto que ella es la víctima principal de las acciones de los hombres que la rodean. Con todo, resulta sumamente interesante darse cuenta de que Kon Ichikawa la presenta hasta en tres ocasiones en un plano frontal dentro de los confines de la casa y enfrentada a los hombres que definirán su destino: a Nobuchi al principio del filme, a Kaji y Nobuchi cuando se mudan a la casa de su madre $y$, finalmente, a Hioki cuando acude al funeral de su marido. La circularidad narrativa se instaura así y propicia la aparición de la repetición que permite interpretar la presencia de Hioki dentro de las paredes de su hogar como prueba de que asumirá el rol de Sensei.

\section{CONCLUSIÓN}

A lo largo de este artículo hemos intentado subrayar el debate discursivo que se instaura entre el Kokoro escrito por Natsume Sōseki y la adaptación cinematográfica de Kon Ichikawa. Los trasvases y las reinterpretaciones son 
constantes, pero, quizá, lo más destacable de la versión del cineasta japonés sea su capacidad para entablar un diálogo con las distintas corrientes literarias que han analizado la novela. Inmerso en un debate sobre las posibles implicaciones y significados ocultos del texto original que sigue vigente, Ichikawa también optó por posicionarse en el terreno del tabú y de la controversia para dar vida a su Kokoro. En un contexto en el que la lectura nostálgica de Jun Etō empezaba a establecerse como la norma, la película se desviste de la carga histórica que explora Sōseki -aunque sin omitirla-para centrarse en el mundo interior de sus personajes. Los silencios que tiñen la prosa en el original, se convierten aquí en una sucesión de primeros planos que intentan penetrar en la psique de sus protagonistas, que pretenden decir con una mirada a cámara todo lo que ocultan sus rostros. El espíritu de Kokoro se preserva parcialmente con esta planificación desnuda de artificios, pero, a pesar del enorme talento de su elenco, la simplificación de la trama obliga a la psicología de los personajes a cambiar irremediablemente, fundamentalmente en el caso de Nobuchi, que pierde complejidad y matices a medida que la película va arrancando y omitiendo episodios vitales de su pasado. La que sí se mantendrá intacta es esa sensación de que Sensei se asfixia en su propia piel, de que se siente atrapado. No solamente porque el ahogamiento sea la metáfora principal del filme ya desde los títulos de crédito iniciales, sino por la circularidad que se establece en torno a este leit motiv (de un modo similar a como hace con el personaje de Shizu) para expresar las trágicas consecuencias de la tensión homoerótica primero en el mar (para introducir la relación entre Nobuchi y Hioki) y posteriormente en el desierto (como premonición de ese entorno social que terminará por aniquilar el vínculo entre Kaji y Nobuchi).

En el centro de este caos emocional se sitúa a Shizu, esposa abnegada y sufriente, que se convierte en la única fuente de luz del largometraje. A diferencia de la novela, donde la joven permanece ajena a todo el drama desatado en el corazón de su marido, la Shizu de la película sí da muestras que comprender la tragedia que ha empañado su matrimonio. En un mundo de claroscuros fílmicos, donde Nobuchi se ve situado repetidamente en la sombra y su mujer en la luz - contraste ya establecido desde la primera escena en la que el matrimonio comparte encuadre-, Kon Ichikawa se apoyará en la iluminación y en los primeros planos para plasmar en celuloide lo que sus personajes no osan decir con palabras. Los remordimientos de Sensei, el sufrimiento de Shizu, la fascinación de Hioki, la pueril determinación de Kaji y, por encima de todo, la culpa y la crueldad de Nobuchi se van sumando y superponiendo para construir un caleidoscopio de claroscuros donde cada nueva imagen, cada nueva mirada sostenida, cada gesto de dolor intenta revelar una faceta de ese complejo kokoro concebido 
por Natsume Sōseki pero que no se recrea, como en el caso del novelista, en la representación del mundo cambiante de la era Meiji sino en el anhelo individual de sus protagonistas.

\section{BIBLIOGRAFÍA CITADA}

ETŌ, Jun (1965), «Natsume Sōseki: A Japanese Meiji Intellectual», The American Scholar, 34/4, otoño, págs. 603-619.

KaWAna, Sari (2018), The Uses of Literature in Modern Japan, Londres, Bloomsbury Publishing.

MCKinney, Meredith (2010), «Introduction» a Natsume Sōseki, Kokoro, NuevaYork, Penguin Books.

MCLellond, Mark J. (2000), Male Homosexuality in Modern Japan: Cultural Myths and Social Realitles, Richmond, Curzon.

Mostow, Joshua (ed.) (2003), The Columbia Companion to Modern East Asian Literature, Nueva York, Columbia University Press.

NaITO, Mariko (2010), «Rethinking the Dichotomy between the Religious and the Secular: The Emergence of Religion in Modern Japan», UTCP Booklet, 17, diciembre, págs. 23-31. [En línea: https://utcp.c.utokyo.ac.jp/publications/pdf/UTCPBooklet17 01 Naito.pdf].

NaKano, Tsutomu (2018), Japanese Management in Evolution: New Directions, Breaks, and Emerging Practices, Nueva York, Routledge.

Nathan, John (2018), Sōseki: Modern Japan's Greatest Novelist, Nueva York, Columbia University Press.

RuBio, Carlos (2009), «Introducción» a Natsume Sōseki, Kokoro, Barcelona, Gredos [edición en formato electrónico].

Ryans, Tony (2009), Kokoro Booklet (DVD), London, Eureka/The Masters of Cinema Series 67.

SHARP, Jasper (2011), Historical Dictionary of Japanese Cinema, Plymouth, The Scarecrow Press.

SHARP, Jasper (2018), «Where to begin with Kon Ichikawa» [En línea: https://www.bfi.org.uk/news-opinion/news-bfi/features/wherebegin-kon-ichikawa].

SōSEKI, Natsume (2018), Kokoro, Madrid, Impedimenta.

STANDISH, Paul (2012), «Sounding the Echoes. By Way of an Introduction», en P. Standish y S. Naoko (eds.), Education and the Kyoto School of Philosophy: Pedagogy for Human Transformation, Nueva York, Springer, págs. 1-15.

VINCENT, J. Keith (2010), «Sexuality and narrative in Sōseki's Kokoro», en N. Cornyetz y J. K. Vincent (eds.), Perversion and Modern Japan: Psychoanalysis, Literature, Culture, Nueva York, Routledge, págs. 222240. 
Claroscuros del corazón: Kokoro

Fecha de recepción: 27/08/2019.

Fecha de aceptación: 25/09/2019. 